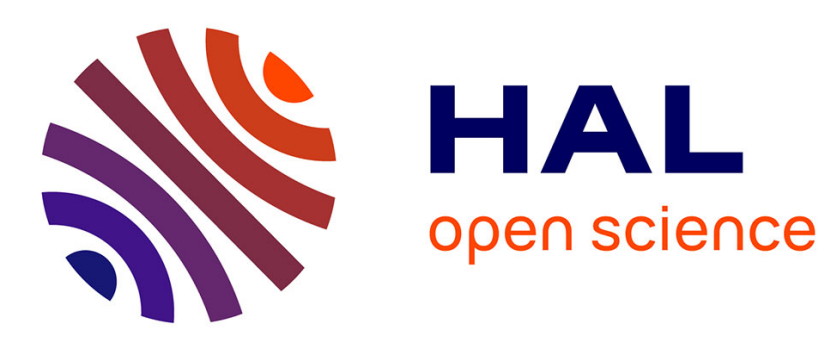

\title{
Resource Block-Level Power Allocation in Asynchronous Multi-Carrier D2D Communications
}

Mylène Pischella, Rostom Zakaria, Didier Le Ruyet

\section{To cite this version:}

Mylène Pischella, Rostom Zakaria, Didier Le Ruyet. Resource Block-Level Power Allocation in Asynchronous Multi-Carrier D2D Communications. IEEE Communications Letters, 2017, 21 (4), pp.813816. 10.1109/LCOMM.2016.2640273 . hal-02446313

\section{HAL Id: hal-02446313 https://hal-cnam.archives-ouvertes.fr/hal-02446313}

Submitted on 20 Mar 2020

HAL is a multi-disciplinary open access archive for the deposit and dissemination of scientific research documents, whether they are published or not. The documents may come from teaching and research institutions in France or abroad, or from public or private research centers.
L'archive ouverte pluridisciplinaire HAL, est destinée au dépôt et à la diffusion de documents scientifiques de niveau recherche, publiés ou non, émanant des établissements d'enseignement et de recherche français ou étrangers, des laboratoires publics ou privés. 


\title{
Resource Block level power allocation in asynchronous multi-carrier D2D communications
}

\author{
Mylene Pischella, Rostom Zakaria and Didier Le Ruyet, Senior Member, IEEE
}

\begin{abstract}
This letter focuses on weighted sum rate maximization with Filter Bank Multi-Carrier (FBMC) and Orthogonal Frequency Division Multiplex (OFDM) multi-carrier modulations for asynchronous Device-to-Device (D2D) communications. The main difficulty in power allocation with asynchronous multicarrier transmissions is that inter-channel interference (ICI) depends on subcarriers, whereas power values should be optimized with Resource Block (RB) granularity. In this letter, we show that the weighted sum rate maximization problem can be solved at RB level, while still taking into account ICI, and that the power allocation algorithm solving this problem is distributed and leads to its global optimum. Moreover, FBMC achieves higher data rates than OFDM, thanks to its lower ICI spread.
\end{abstract}

\section{INTRODUCTION}

Achieving high data rates with asynchronous transmissions is one of the main technical challenges of future $5 \mathrm{G}$ systems. 5G will be based on new multi-carrier techniques that should handle asynchronicity. Filter Bank Multi-Carrier (FBMC) is well-localized in the frequency domain, leading to better performance results than Orthogonal Frequency Division Multiplex (OFDM) in most asynchronous scenarios [1], [2]. In previous literature, resource allocation in asynchronous transmissions has always been performed at the subcarrier level. However in practice, resource allocation is performed at the Resource Block (RB) level [3], involving several adjacent subcarriers. Inter-channel interference (ICI) generated by asynchronous transmissions cannot be integrated or averaged on RBs. As a consequence, deriving resource allocation algorithms at the RB level for multi-carrier asynchronous transmissions is still an open issue.

In this letter, we focus on the weighted sum rate maximization problem in asynchronous Device-to-Device (D2D) communications, contrary to prior work on D2D resource allocation that assumed full synchronicity [4]-[7]. D2D pairs may be multiplexed on the same RBs if they are distant enough. A maximum interference constraint per subcarrier at the Base Station (BS) is also added. This constraint expresses that D2D transmitters, that are active in the uplink (UL) according to LTE standard [3], should not disturb cellular users communications. The weighted sum maximization objective covers a large range of resource allocation issues: cell sum rate maximization, proportional fair, or throughput optimal transmissions, when users weights are proportional to their queue lengths. Therefore, this letter's contribution is a first

The authors are with CNAM CEDRIC/LAETITIA, 292 rue Saint-Martin, 75003 Paris, France (contact: mylene.pischella@cnam.fr). This work was partially funded through French National Research Agency (ANR) project ACCENT5 with grant agreement code: ANR-14-CE28-0026-02. step to RB level resource allocation for 5G D2D asynchronous transmissions. A resource allocation algorithm is determined in section III and a distributed implementation is proposed. Finally, FBMC and OFDM are compared through simulations in section IV.

\section{SYSTEM MODEL}

We consider $K$ D2D pairs located in one cell. A frequency reuse factor (FRF) of at least 3 is used, so that resource allocation can be made per cell while assuming that intercell interference is negligible. OFDM and FBMC multi-carrier techniques with $N$ RBs composed of $M$ adjacent subcarriers are compared. Let $L=M \times N$ be the total number of subcarriers. The optimization objective is to maximize the sum of utility functions $u_{k}^{r}(\mathbf{p})=\alpha_{k} \log \left(1+\operatorname{SINR}_{k}^{r}\right)$ where $\alpha_{k}$ is the weight of user $k$ and $\operatorname{SINR}_{k}^{r}$ is the Signal to Interference plus Noise Ratio (SINR) of user $k$ in $\mathrm{RB} r$ and $\mathbf{p}$ is the power vector. To simplify notations, in the following, $\log (x)$ stands for $\log _{2}(x)$. We assume that RBs allocation has been performed before power allocation. RBs are allocated to subsets of distant D2D pairs that generate low interference to each others. Thanks to this RB allocation, to the FRF, and to low distances between transmitters and receivers of the same D2D pairs, the high SINR assumption holds. The utility function per user and $\mathrm{RB}$ is then simplified to: $u_{k}^{r}(\mathbf{p})=\alpha_{k} \log \left(\operatorname{SINR}_{k}^{r}\right)$.

All D2D transmitters are synchronous with their receiver, and asynchronous with any other receiver, including the BS. Then, each D2D transmitter's power in RB $r$ generates ICI at the other D2D receivers, not only on the subcarriers of $\mathrm{RB}$ $r$, but also on the subcarriers of the adjacent RBs. The ICI is modelled as interference weights to apply on the power vector $\mathbf{p}$ [8]. Their spread and amplitude depend on the multi-carrier modulation type. Let $\Delta$ be the OFDM cyclic prefix $(\mathrm{CP})$ and $T$ the multi-carrier symbol durations. The ICI weights expressions have been derived in [8] for OFDM and FBMC, when the timing offset is uniformly distributed in $[0 ; T+\Delta]$ and $[0 ; T]$, respectively. Each subcarrier $l$ generates ICI weights on at most $D$ adjacent subcarriers $l^{\prime}$ on its left and right. $D$ depends on the multi-carrier modulation and is larger with OFDM than with FBMC. ICI weights are gathered in vector $\mathbf{V}$ of size $L$, where $V_{\left|l-l^{\prime}\right|}=0$ if $\left|l-l^{\prime}\right|>D$.

Let us denote by $\overline{\mathbf{G}}_{k q}$ the $L \times L$ channel gain matrix between the transmitter of the $q$-th D2D pair and the receiver of the $k$-th D2D pair. The elements of the matrix $\overline{\mathbf{G}}_{k q}$ are given by:

$$
\left\{\begin{array}{l}
\overline{\mathbf{G}}_{k q}(i, j)=g_{k q}(j) V_{|i-j|} \quad \forall k \neq q \\
\overline{\mathbf{G}}_{k k}(i, j)=g_{k k}(j) \delta_{i-j} \quad \forall k
\end{array}\right.
$$


where $g_{k q}(j)$ is the channel gain from the transmitter of pair $q$ to the receiver of pair $k$ in the $j$-th subcarrier, $V_{d}$ is the interference coefficient for the spectral distance $d \geq 0$, and $\delta_{x}$ stands for the Kronecker delta. Let $p_{q}(j)$ be the transmitted power at the $j$-th subcarrier by the transmitter $q$. Then the vector $\overline{\mathbf{y}}_{k}$ composed of received signal powers $y_{k}(l)$ at the $l$-th subcarrier for the $k$-th receiver, $\forall k \in\{0, \ldots, K-1\}$, is:

$\underbrace{\left[\begin{array}{c}y_{k}(0) \\ \vdots \\ y_{k}(L-1)\end{array}\right]}_{\overline{\mathbf{y}}_{k}}=\overline{\mathbf{G}}_{k k} \underbrace{\left[\begin{array}{c}p_{k}(0) \\ \vdots \\ p_{k}(L-1)\end{array}\right]}_{\overline{\mathbf{p}}_{k}}+\sum_{\substack{q=0 \\ q \neq k}}^{K-1} \overline{\mathbf{G}}_{k q} \underbrace{\left[\begin{array}{c}p_{q}(0) \\ \vdots \\ p_{q}(L-1)\end{array}\right]}_{\overline{\mathbf{p}}_{q}}$

Let $\tilde{\mathbf{p}}=\left[\overline{\mathbf{p}}_{0}^{T}, \overline{\mathbf{p}}_{1}^{T}, \ldots, \overline{\mathbf{p}}_{K-1}^{T}\right]^{T} \quad$ and $\quad \mathbf{y}=$ $\left[\overline{\mathbf{y}}_{0}^{T}, \overline{\mathbf{y}}_{1}^{T}, \ldots, \overline{\mathbf{y}}_{K-1}^{T}\right]^{T}$. Equation (2) can be written as:

$$
\mathbf{y}=\underbrace{\left[\begin{array}{cccc}
\overline{\mathbf{G}}_{00} & \overline{\mathbf{G}}_{01} & \ldots & \overline{\mathbf{G}}_{0(K-1)} \\
\overline{\mathbf{G}}_{10} & \overline{\mathbf{G}}_{11} & \ldots & \overline{\mathbf{G}}_{1(K-1)} \\
\vdots & & \ddots & \vdots \\
\overline{\mathbf{G}}_{(K-1) 0} & \overline{\mathbf{G}}_{(K-1) 1} & \ldots & \overline{\mathbf{G}}_{(K-1)(L-1)}
\end{array}\right]}_{\tilde{\mathbf{G}}} \tilde{\mathbf{p}}
$$

In this letter, we assume that power allocation is performed at RB level. Consequently, all subcarriers within a single RB have the same allocated power. In this case, the vector of transmit power $\tilde{\mathbf{p}}$ is expressed as:

$$
\tilde{\mathbf{p}}=\left(\mathbf{I}_{K N} \otimes \mathbf{1}_{M}\right) \mathbf{p}
$$

where $\otimes$ stands for the Kronecker product, $\mathbf{I}_{K N}$ is the identity matrix of size $K N, \mathbf{1}_{M}$ is a $M \times 1$ vector whose elements are set to 1 , and $\mathbf{p}$ is a $K N \times 1$ vector whose entries are the transmit power in each RB. Then eq. (3) becomes:

$$
\mathbf{y}=\tilde{\mathbf{G}} \underbrace{\left(\mathbf{I}_{K N} \otimes \mathbf{1}_{M}\right)}_{\mathbf{S}} \mathbf{p}=\mathbf{F} \mathbf{p}
$$

where $\mathbf{F}=\tilde{\mathbf{G}} \mathbf{S}$ is a $K L \times K N$ matrix.

Similarly, the interference received by the BS from D2D transmitters is written as:

$$
\begin{aligned}
\mathbf{I}_{B S} & =\underbrace{\left[\begin{array}{llll}
\overline{\mathbf{A}}_{0} & \overline{\mathbf{A}}_{1} & \ldots & \overline{\mathbf{A}}_{K-1}
\end{array}\right]}_{\tilde{\mathbf{A}}} \tilde{\mathbf{p}} \\
& =\tilde{\mathbf{A}} \mathbf{S p}=\mathbf{A} \mathbf{p}
\end{aligned}
$$

where $\overline{\mathbf{A}}_{k}$ is a $L \times L$ matrix given by:

$$
\overline{\mathbf{A}}_{k}(i, j)=h_{k}(j) V_{|i-j|} \forall k \in\{0, \ldots, K-1\}
$$

and $h_{k}(j)$ is the interference channel gain at subcarrier $j$ between the $k$-th D2D tranmitter and the BS.

The following notations are used in the rest of the letter:

- $P_{j}^{r}=\mathbf{p}(r+j M)$ is the power allocated for user $j$ in the $r$-th RB,

- $F_{r j}^{l k}=\mathbf{F}(l+k L, r+j N)$ is the element of matrix $\mathbf{F}$ in line $l+k L$ and row $r+j N$,

- $A_{k r}^{l}=\mathbf{A}(l, r+k N)$ is the interference gain at the BS in subcarrier $l$ from transmitter $k$ and RB $r$,

- $F_{r k}^{l k}$ is the direct channel between transmitter $k$ and its receiver in subcarrier $l$,
- $\mathbb{B}_{j}$ is the set of RB indices used by transmitter $j$,

- $\mathbb{R}_{r}$ is the index set of the subcarriers in the $r$-th RB.

The interference received by user $k$ in subcarrier $l I_{k}^{l}$ is then:

$$
I_{k}^{l}=\sum_{\substack{j=0 \\ j \neq k}}^{K-1} \sum_{r \in \mathbb{B}_{j}} F_{r j}^{l k} P_{j}^{r}
$$

and the utility function per user $k$ and $\mathrm{RB} r$ becomes:

$$
\begin{aligned}
u_{k}^{r}(\mathbf{p}) & =\sum_{l \in \mathbb{R}_{r}} \alpha_{k} \log \left(\frac{F_{r k}^{l k} P_{k}^{r}}{n_{k}^{l}+I_{k}^{l}}\right) \\
& =\alpha_{k} M \log \left(P_{k}^{r}\right)+\alpha_{k} \log \left(\prod_{l \in \mathbb{R}_{r}} \frac{F_{r k}^{l k}}{n_{k}^{l}+I_{k}^{l}}\right)
\end{aligned}
$$

\section{Power Allocation for the Weighted SUM RATE MAXIMIZATION PROBLEM}

The weighted sum rate maximization problem is written as:

$$
\begin{aligned}
& \max _{\mathbf{p} \geq 0} \sum_{k=0}^{K-1} \sum_{r \in \mathbb{B}_{k}} u_{k}^{r}(\mathbf{p}) \\
& \text { s.t. } M \sum_{r \in \mathbb{B}_{k}} P_{k}^{r} \leq P_{\max } \forall k \in\{0, \ldots, K-1\} \\
& \text { s.t. } \sum_{k=0}^{K-1} \sum_{r \in \mathbb{B}_{k}} A_{k r}^{l} P_{k}^{r} \leq I_{0} \forall l \in\{0, \ldots, L-1\}
\end{aligned}
$$

where $P_{\max }$ is the maximum transmit power per user and $I_{0}$ is the maximum allowed interference per subcarrier at the BS. Since problem (10) belongs to the class of geometric programming [9], it has a unique optimal solution which must satisfy the Karush-Kuhn-Tucker (KKT) conditions. The Lagrangian of problem (10) is:

$$
\begin{aligned}
\mathcal{L}(\mathbf{p}) & =\sum_{k=0}^{K-1} \sum_{r \in \mathbb{B}_{k}} u_{k}^{r}(\mathbf{p})-\sum_{k=0}^{K-1} \mu_{k}\left(M \sum_{r \in \mathbb{B}_{k}} P_{k}^{r}-P_{\max }\right) \\
& -\sum_{l=0}^{L-1} \lambda_{l}\left(\sum_{k=0}^{K-1} \sum_{r \in \mathbb{B}_{k}} \frac{A_{k r}^{l}}{I_{0}} P_{k}^{r}-1\right)
\end{aligned}
$$

where $\boldsymbol{\mu}$ and $\boldsymbol{\lambda}$ are Lagrange multipliers, that are positive by definition. At the optimal solution $\mathbf{p}^{*}$, the gradient of the Lagrangian is equal to $\mathbf{0}$. Then there exists unique Lagrange multiplier vectors $\boldsymbol{\mu}^{*}$ and $\boldsymbol{\lambda}^{*}$ such that for all $k_{0}$ and $r_{0} \in \mathbb{B}_{k_{0}}$ :

$$
\frac{\alpha_{k_{0}} M}{P_{k_{0}}^{r_{0} *}}+\sum_{\substack{j=0 \\ j \neq k_{0}}}^{K-1} \sum_{r \in \mathbb{B}_{j}} \frac{\partial u_{j}^{r}\left(\mathbf{p}^{*}\right)}{\partial P_{k_{0}}^{r_{0}}}=\mu_{k_{0}} M+\sum_{l=0}^{L-1} \frac{A_{k_{0} k_{0}}^{l}}{I_{0}} \lambda_{l}
$$

The derivative of utility functions $u_{j}^{r}$ with respect to $P_{k_{0}}^{r_{0}}$, with $j \neq k_{0}$, is:

$$
\frac{\partial u_{j}^{r}\left(\mathbf{p}^{*}\right)}{\partial P_{k_{0}}^{r_{0}}}=-\alpha_{j} \sum_{l \in \mathbb{R}_{r}} \frac{F_{r_{0} k_{0}}^{l j}}{n_{j}^{l}+I_{j}^{l}}
$$

Consequently, equation (12) becomes:

$$
\frac{\alpha_{k_{0}} M}{P_{k_{0}}^{r_{0} *}}-\sum_{\substack{j=0 \\ j \neq k_{0}}}^{K-1} \sum_{r \in \mathbb{B}_{j}} \sum_{l \in \mathbb{R}_{r}} \frac{\alpha_{j} F_{r_{0} k_{0}}^{l j}}{n_{j}^{l}+I_{j}^{l}}=\mu_{k_{0}} M+\sum_{l=0}^{L-1} \frac{A_{k_{0} r_{0}}^{l}}{I_{0}} \lambda_{l}
$$


And the optimum value of $P_{k_{0}}^{r_{0}}$ finally is:

$$
P_{k_{0}}^{r_{0} *}=\frac{\alpha_{k_{0}} M}{f_{k_{0}}^{r_{0}}\left(\mathbf{p}^{*}, \mu_{k_{0}}^{*}, \boldsymbol{\lambda}^{*}\right)}
$$

with

$$
\begin{aligned}
& f_{k_{0}}^{r_{0}}\left(\mathbf{p}^{*}, \mu_{k_{0}}^{*}, \boldsymbol{\lambda}^{*}\right)=\mu_{k_{0}}^{*} M+\sum_{l=0}^{L-1} \frac{A_{k_{0} r_{0}}^{l}}{I_{0}} \lambda_{l}^{*} \\
& +\sum_{\substack{j=0 \\
j \neq k_{0}}} \sum_{l \in \Omega_{j}} \frac{\alpha_{j} F_{r_{0} k_{0}}^{l j}}{n_{j}^{l}+I_{j}^{l}\left(\mathbf{p}^{*}\right)}
\end{aligned}
$$

where $\Omega_{j}=\bigcup_{r \in \mathbb{B}_{j}} \mathbb{R}_{r}$ is the set of subcarriers allocated to user $j$.

We now prove that the power allocation algorithm can be implemented in a distributed way. We notice from (5) that

$$
\mathbf{F}(i, j)=\sum_{k=0}^{K L-1} \tilde{\mathbf{G}}(i, k) \mathbf{S}(k, j)=\sum_{k=j M}^{j M+(M-1)} \tilde{\mathbf{G}}(i, k)
$$

since $\mathbf{S}(k, j)=1$ if $\left\lfloor\frac{k}{j}\right\rfloor=j$ and $\mathbf{S}(k, j)=0$ elsewhere, with $\lfloor x\rfloor$ the nearest lower integer of $x$.

Besides, by construction of $\tilde{\mathbf{G}}$ (see eq. (3)) :

$$
\tilde{\mathbf{G}}(i, k)=\overline{\mathbf{G}}_{\left\lfloor\frac{i}{L}\right\rfloor\left\lfloor\frac{k}{L}\right\rfloor}\left(i-\left\lfloor\frac{i}{L}\right\rfloor L, k-\left\lfloor\frac{k}{L}\right\rfloor L\right)
$$

Then $F_{r_{0} k_{0}}^{l j}$ is equal to:

$$
F_{r_{0} k_{0}}^{l j}=\mathbf{F}\left(l+j L, r_{0}+k_{0} N\right)=\sum_{p=0}^{M-1} \overline{\mathbf{G}}_{j k_{0}}\left(l, r_{0} M+p\right)
$$

Using the initial definition (1) of $\overline{\mathbf{G}}$, we finally obtain:

$$
F_{r_{0} k_{0}}^{l j}=\sum_{p=0}^{M-1} g_{j k_{0}}\left(r_{0} M+p\right) V_{\left|l-r_{0} M-p\right|} \quad \forall j \neq k_{0}
$$

Equation (18) shows that a distributed iterative algorithm can be used for power allocation. It requires a downlink (DL) and an UL control channel, where D2D transmitters and receivers are synchronized with the BS. The following implementation is proposed: in an initial phase, each D2D transmitter sends a low-length pilot signal on the whole bandwdith of the data channel in a dedicated time slot. Since only one transmitter $k_{0}$ is active per time slot, channel gains $g_{j k_{0}}(l), l \in\{0, \ldots, L-1\}$ can be estimated by each receiver $j$ and $h_{k_{0}}(l)$ by the BS after time synchronization. After all transmitters have sent their pilot signal, each receiver $j$ sends $g_{j k}(l), \forall k \neq j$, to the BS on the UL control channel. The BS forwards them to all transmitters on the DL control channel, as well as $I_{0}$ and $h_{k}(l), \forall k$. Knowing the constant vector $\mathbf{V}$, transmitter $k_{0}$ computes $A_{k_{0} r_{0}}^{l}$ with eq. (7) and $F_{r_{0} k_{0}}^{l j}$ with eq. (??). Afterwards, during the iterative phase, each receiver $j$ transmits interference information $\Pi_{j}^{l}(\mathbf{p})$ to the BS on the UL control channel:

$$
\Pi_{j}^{l}(\mathbf{p})=\frac{\alpha_{j}}{n_{j}^{l}+I_{j}^{l}(\mathbf{p})}
$$

The BS then forwards all $\Pi_{j}^{l}(\mathbf{p})$ to all D2D transmitters on the DL control channel, along with $\boldsymbol{\lambda}$ if its value has been updated. Transmitter $k_{0}$ finally computes $\sum_{l \in \Omega_{j}} F_{r_{0} k_{0}}^{l j} \Pi_{j}^{l}(\mathbf{p})$ in RB $r_{0}$ and updates $P_{k_{0}}^{r_{0}}$ with (14).

To summarize, the power allocation algorithm is performed as follows: first, at $T=0$, dual prices $\mu_{k_{0}}(T), \forall k_{0} \in$ $\{0, \ldots, K-1\}$ and $\lambda_{l_{0}}(T), \forall l_{0} \in\{0, \ldots, L-1\}$ are initialized. Then for fixed values of $\boldsymbol{\mu}(T)$ and $\boldsymbol{\lambda}(T)$, an iterative algorithm is used for power allocation, independently on all RB $r_{0} \in\{0, \ldots, N-1\}$. $\mathbf{p}$ and $\Pi$ are initialized with equal power allocation. Then at each iteration $T_{i}<T_{i, \max }$, where $T_{i, \max }$ is the maximum number of iterations, all users $k_{0}$ active in $r_{0}$ perform the following two steps:

- Power update: use eq. (14) with $\mathbf{p}\left(T_{i}\right), \mu_{k_{0}}(T)$ and $\boldsymbol{\lambda}(T)$ as inputs to compute $\mathbf{p}\left(T_{i}+1\right)$.

- Interference information update: compute $\Pi_{k_{0}}^{l_{0}}\left(\mathbf{p}\left(T_{i}+1\right)\right)$ from eq. (19).

At the end of this loop, dual prices are updated as follows, where $\kappa>0$ and $\delta>0$ are small positive steps:

$\mu_{k_{0}}(T+1)=\left[\mu_{k_{0}}(T)+\kappa\left(\sum_{r \in \mathbb{B}_{k_{0}}} M P_{k_{0}}^{r}\left(T_{i, \max }\right)-P_{\max }\right)\right]^{+}$

$\forall k_{0} \in\{0, \ldots, K-1\}$, where $[a]^{+}=\max \{0, a\}$, and

$\lambda_{l_{0}}(T+1)=\left[\lambda_{l_{0}}(T)+\delta\left(\sum_{k=0}^{K-1} \sum_{r \in \mathbb{B}_{k}} \frac{A_{k r}^{l_{0}}}{I_{0}} P_{k}^{r}\left(T_{i, \max }\right)-1\right)\right]^{+}$

$\forall l_{0} \in\{0, \ldots, L-1\}$. This process is repeated $T_{d, \max }$ times until convergence.

Similarly to [10], it can be shown that this algorithm converges for small enough values of steps $\kappa>0$ are $\delta>0$. Since the fixed point of the iterative algorithm verifies all KKT conditions of problem (10), it is its global optimum. The total number of operations of the algorithm is:

$$
\begin{aligned}
\mathcal{C} & =T_{d, \max }[K N+L K(2 D+1) \\
& \left.+T_{i, \max }\left(K N L+K^{2} N(2 D+1)+L K\right)\right]
\end{aligned}
$$

The algorithm's complexity is polynomial in $K, L$ and $N$, and is thus feasible in practice with reasonable processing delay. We can also notice that the computational complexity is far lower than with per-subcarrier power allocation. For instance, without constraint $(C 2)$ in (10) and with PS, if $K=20$, it is 7.9 times lower if $M=12$ than if $M=1$.

\section{Simulation RESUlts AND ANALYSis}

Monte-Carlo simulations are conducted with a cell bandwidth $B=10 \mathrm{MHz}$. The Fast Fourier Transform size is 1024. There are $L=600$ active subcarriers of $15 \mathrm{kHz}$ grouped in $N=50 \mathrm{RBs}$ and the cell radius is $500 \mathrm{~m}$ with omnidirectional antenna. D2D transmitters' locations follow a uniform distribution in the cell, and each receiver is uniformly located at most at $50 \mathrm{~m}$ from its transmitter. $P_{\max }$ is equal to $21 \mathrm{dBm}$ and the thermal noise per subcarrier is equal to -132 $\mathrm{dBm}$. Each D2D pair has a uniform random weight between 0 and 1 , with $\sum_{k=1}^{K} \alpha_{k}=1$. The path loss model is small cells $L_{\mathrm{dB}}=140+36.8 \log _{10}(d)$ if the receiver is a device, and LTE urban $L_{\mathrm{dB}}=128.1+37.6 \log _{10}(d)$ if the receiver is the BS. The log-normal shadowing has a standard deviation equal to 
$4 \mathrm{~dB}$ for D2D communications and to $9 \mathrm{~dB}$ for Device-toBS interference. Multi-path fading is computed with Indoor Channel-B model [11].

ICI weights are computed using the formula from [8] with OFDM LTE parameters $\Delta=4.69 \mu \mathrm{s}$ and $T=66.6 \mu \mathrm{s}$. Only weights exceeding $10^{-3}$ are considered. Then $D$ is equal to 9 with OFDM and 1 with FBMC. The $D+1$ non-zero elements of vector $\mathbf{V}$ are equal to:

$$
\begin{aligned}
\mathbf{V}_{\text {OFDM }} & =\left[6.89 \times 10^{-1}, 9.47 \times 10^{-2}, 2.37 \times 10^{-2}\right. \\
& 1.05 \times 10^{-2}, 5.9 \times 10^{-3}, 3.8 \times 10^{-3} \\
& \left.2.6 \times 10^{-3}, 1.9 \times 10^{-3}, 1.5 \times 10^{-3}, 1.12 \times 10^{-3}\right] \\
\mathbf{V}_{\text {FBMC }} & =\left[8.23 \times 10^{-1}, 8.81 \times 10^{-2}\right]
\end{aligned}
$$

The reference vector is $\mathbf{V}_{\mathrm{PS}}=[1]$ for Perfectly Synchronized (PS) transmission, which represents a theoretical upper-boud with $\mathrm{CP} \Delta=0$.

RB allocation is performed by graph-coloring with DSATUR algorithm [12]: if the distance between transmitter $k$ and receiver $k^{\prime}$, with $k \neq k^{\prime}$, is lower than a threshold $D_{\text {int }}, k$ and $k^{\prime}$ belong to different colors. $D_{\text {int }}$ is obtained by bisection search to exactly reach 5 colors, whatever the number of D2D pairs in the cell. Then all D2D pairs of color $c$ are multiplexed on $\mathrm{RBs} l$ such that $l \bmod (5)=c$. RBs are spread in the bandwidth in order to benefit from frequency diversity.

Fig. 1 shows the performance of our proposed algorithm (called DADP for Dual Asynchronous Distributed Pricing) when constraint $(C 2)$ is not taken into account. It is compared with a reference case without power control (called EPA for Equal Power Allocation), where each D2D transmitter equally splits its power on all its allocated RB. Fig. 1 shows the effectiveness of DADP: with FBMC, the weighted sum rate with DADP is up to $9.4 \%$ higher than that achieved with EPA, which corresponds to an increase of $1.5 \mathrm{Mbits} / \mathrm{s}$. Besides, the weighted sum rate with DADP-FBMC is very close to that obtained with DADP-PS (its decrease is at most $2.5 \%$ ), contrary to OFDM (its decrease reaches $14.8 \%$ ). This is due to the lower ICI spread of FBMC and the CP of OFDM, that generates an overhead of $\Delta /(\Delta+T)$. Fig. 2 represents the weighted sum rate with DADP when $K=32$ with constraint $(C 2)$, when $I_{0}$ varies. It is not possible to compare with EPA in this case since EPA would not necessarily fulfill constraint $(C 2)$. FBMC is still far more efficient than OFDM, and the weighted sum rate with FBMC is less than $1.8 \%$ from the upper bound obtained with PS. Moreover, we can notice that D2D data rates are very high, even though they generate low interference level at the BS. This shows that D2D pairs can be efficiently underlaid in cellular networks.

\section{CONCLUSIONS}

In this letter, the weigthed sum rate maximization of D2D users with asynchronous transmissions has been studied. Even though ICI weights are defined at subcarrier level, we showed that RB level optimization is feasible and that the corresponding power allocation algorithm is distributed. These two features are very useful for practical implementations in future $5 \mathrm{G}$ networks. Besides, simulations results showed the superiority of FBMC over OFDM in asynchronous transmissions.

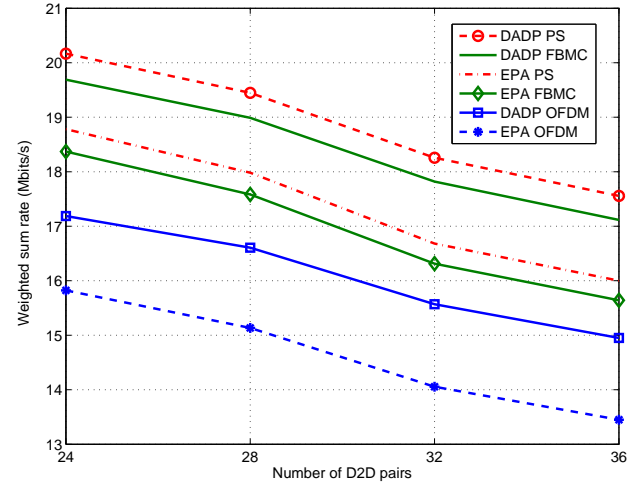

Fig. 1. Weighted sum rate vs number of D2D pairs

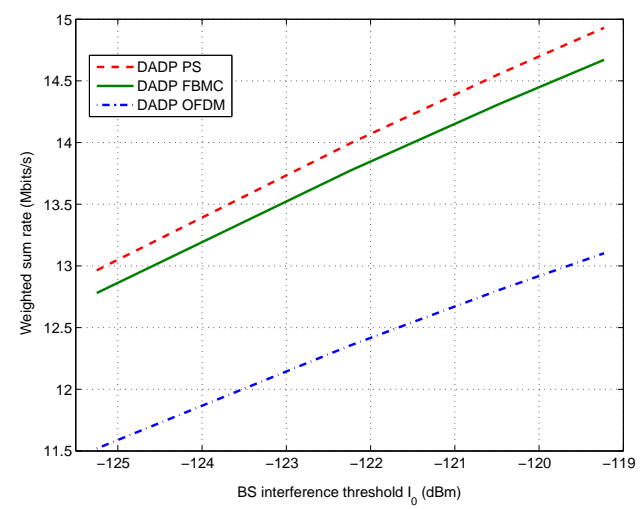

Fig. 2. Weighted sum rate vs $I_{0}, K=32$

\section{REFERENCES}

[1] M. G. Bellanger, "Specification and Design of a Prototype Filter for Filter Bank based Multicarrier Transmission," in Proc. of ICASSP 2001, Salt Lake City, UT, USA, May 2001.

[2] M. Shaat and F. Bader, "Computationally efficient power allocation algorithm in multicarrier-based cognitive radio networks: OFDM and FBMC systems," EURASIP Journal on Advances in Signal Processing, vol. 2010, Article ID 528378, 2010.

[3] “3GPP TR 36.843 V1.0.1, 3rd Generation Partnership Project; Technical Specification Group Radio Access Network; Study on LTE Device to Device Proximity Services; Radio Aspects (Release 12)," Feb 2014.

[4] P. Mach, Z. Becvar, and T. Vanek, "In-band Device-to-Device communication in OFDMA cellular networks: a survey and challenges," IEEE Comm. Surveys and Tutorials, vol. 17, no. 4, pp. 1885-1922, Oct. 2015.

[5] A. Antonopoulos, E. Kartsakli, and C. Verikoulis, "Game Theoreric D2D content dissemination in 4G cellular networks," IEEE Comm. Magazine, vol. 52, no. 6, pp. 125-132, June 2014.

[6] T.D. Hoang, L.B. Le, and T. Le-Ngoc, "Joint subchannel and power allocation for D2D communications in cellular networks," in Proc. of WCNC 2014, Istanbul, Turkey, April 2014.

[7] M. Hasan and E. Hossain, "Distributed resource allocation in D2Denabled multi-tier cellular networks: an auction approach," in Proc. of ICC 2015, London, UK, June 2015.

[8] Y. Medjahdi, M. Terre, D. Le Ruyet, D. Roviras, J. A. Nossek, and L. Baltar, "Inter-cell Interference Analysis for OFDM / FBMC Systems," in Proc. of 10th IEEE Signal Processing Workshop (SPAWC 2009), Perugia, Italy, June 2009.

[9] S. Boyd and L. Vandenbergue, Convex Optimization, Cambridge University Press, 2004.

[10] J. Huang, R. Berry, and M.L. Honig, "Distributed interference compensation for wireless networks," IEEE Jour. Select. Commun., vol. 24, no. 5, pp. 1074-1084, May 2006.

[11] " Guidelines for evaluation of radio transmission technologies for IMT2000," ITU-R Recommendation M.1225, 1997.

[12] D. Brélaz, "New methods to color the vertices of a graph," Communications of the ACM, vol. 22, no. 4, pp. 251-256, Apr. 1979. 